ELECTRONIC RESEARCH ANNOUNCEMENTS OF THE AMERICAN MATHEMATICAL SOCIETY

Volume 11, Pages 57-63 (June 10, 2005)

S $1079-6762(05) 00147-2$

\title{
SPECTRAL AND MIXING PROPERTIES OF ACTIONS OF AMENABLE GROUPS
}

\author{
NIR AVNI
}

(Communicated by Klaus Schmidt)

\begin{abstract}
We generalize two theorems about K-automorphisms from $\mathbb{Z}$ to all amenable groups with good entropy theory (this class includes all unimodular amenable groups which are not an increasing union of compact subgroups). The first theorem is that such actions are uniformly mixing; the second is that their spectrum is Lebesgue with countable multiplicity. For the proof we will develop an entropy theory for discrete amenable equivalence relations.
\end{abstract}

\section{INTRODUCTION}

A probability preserving automorphism $T$ of a probability space $(X, \mathcal{F}, m)$ is called Kolmogorov (or $\mathrm{K}-$ ) automorphism if for every nontrivial partition $P$ of $X, h(T, P)>0$. There are many other characterizations of $\mathrm{K}$-automorphisms, such as being disjoint from all zero entropy transformations and being uniformly mixing. This class also includes all Bernoulli transformations and is a central class in ergodic theory. In $\mathrm{RS}$ it was proved that the spectrum of the unitary operator $U_{T}: L_{2}(X) \rightarrow L_{2}(X)$ given by $U_{T}(f)(x)=f(T x)$ is Lebesgue with countable multiplicity. This theorem was generalized to Kolmogorov flows, that is, one parameter families $\left(T_{t}\right)_{t \in \mathbb{R}}$ for which $T_{1}$ is Kolmogorov, in [S].

In $[\mathrm{OW}]$ (see also $[\mathrm{L}], \overline{\mathrm{W}}], \overline{\mathrm{DP}}]$ ), many results in the ergodic theory of a single transformation (classical ergodic theory) are generalized to amenable groups. Recall that a locally compact second countable group $G$ is called amenable if for every compact $K \subset G$ and for every $\epsilon>0$, there exists a compact $F \subset G$ such that

$$
\lambda(F \triangle K F)<\epsilon \lambda(F) .
$$

In this case we say that $F$ is $(K, \epsilon)$-invariant. We say that a sequence of sets $F_{n}$ becomes more and more invariant if for every $K$ and $\epsilon$, there exists an $N$ such that $F_{n}$ is $(K, \epsilon)$-invariant for all $n>N$. Such a sequence is also called a Følner sequence.

It turns out that the notion of entropy can be generalized to actions of a large class of amenable groups. This class is called groups with good entropy theory, and it includes all unimodular amenable groups which are not an increasing union of compact subgroups and many of those. In particular it includes all unimodular solvable Lie groups. We refer the reader to $\mathrm{OW}$ for the definitions. We denote

Received by the editors May 27, 2004.

2000 Mathematics Subject Classification. Primary 37A15; Secondary 37A20.

(C)2005 American Mathematical Society Reverts to public domain 28 years from publication 
this entropy by $h(G, P)$ when $G$ acts on a probability space and $P$ is a partition (always finite) of that space.

In order to state the results of this paper, we first need to make some further definitions. In the following, assume $G$ is an amenable group with good entropy theory and assume it acts on a probability space $X$ in a measure preserving fashion. We denote by $H(P)$ the entropy of a partition $P$.

Definition 1.1. The action is called completely positive entropy (or CPE for short) if for every nontrivial partition $P$ of $X, h(G, P)>0$.

Definition 1.2. The action is called uniformly mixing if for every partition $P$ of $X$ and every $\epsilon>0$, there exists a compact subset $K \subset G$ such that if $g_{1}, \ldots, g_{n} \in G$ satisfy $g_{i} \notin g_{j} K$ for $i \neq j$, then

$$
\left|H(P)-\frac{1}{n} H\left(g_{1} P \vee g_{2} P \vee \cdots \vee g_{n} P\right)\right|<\epsilon .
$$

Note that if an action is uniformly mixing, then it is mixing of all orders.

Definition 1.3. We say that the action has Lebesgue spectrum with countable multiplicity if $L_{2}(X)$, as a $G$-module, is an orthogonal sum of a countable number of $L_{2}(G)$ 's.

The following is the generalization of the above-mentioned theorem of [RS]. It was proved for discrete amenable groups in [RW] (part a) and [DG] (part b):

Theorem 1.4. Assume $G$ is an amenable group with good entropy theory. Assume that $G$ acts on a probability space $X$ preserving the measure and assume the action is CPE. Then:

a) The action is uniformly mixing, and

b) It has Lebesgue spectrum with countable multiplicity.

The theorem is proved by transferring the result from a certain action of $\mathbb{Z}$ to the action of $G$ on $X$. This transfer has two stages. The first stage is to pass from the continuous group to some discrete structure. More specifically, we pass from the foliation of $X$, where each leaf is isomorphic to $G$, into a foliation of some other space where now each leaf is countable. The second step is to transfer our problem into a problem about an action of $\mathbb{Z}$. This is done by using the theory of orbit equivalence. We now describe more carefully the ideas behind these two steps.

Suppose $(X, \mathcal{F}, m)$ is a probability space and $(r, x) \rightarrow r \cdot x$ is an action of $\mathbb{R}$ on $X$. One strategy of studying this flow is via cross section. A cross section is a Borel subset $A \subset X$ such that for almost every $x \in X$ the set of return times to $A, O_{A}(x)=\{r \in \mathbb{R} \mid r \cdot x \in A\}$ is nonempty and discrete. In this case $O_{A}(x) \cap \mathbb{R}^{+}$ is nonempty for a.e. $x$ and thus contains a minimal element, which we denote by $f(x)$. If we define a transformation $T: A \rightarrow A$ by $T x=f(x) \cdot x$, we have that $T$ preserves a natural probability measure $\mu$ on $A$ and we have Abramov's formula:

$$
h(X, \mathbb{R})=h(A, T) \int_{X} f(x) d \mu(x)
$$

relating the entropies. In this way we reduce the study of actions of $\mathbb{R}$ to the study of a single transformation.

In this article we apply the same idea to actions of amenable groups. We assume from now on that all actions are free, measure preserving and on Borel probability 
spaces 1 When trying to study cross sections of groups different from $\mathbb{R}$, one does not get an action of a discrete subgroup on the cross section, so the construction has to be modified. What one does get naturally is an equivalence relation on the cross section $A: \mathcal{R}=\left\{(x, y) \in A^{2} \mid(\exists g \in G) g x=y\right\}$ and a function $\alpha: \mathcal{R} \rightarrow G$ such that $\alpha(x, y)$ is the (unique) element $g \in G$ such that $g x=y$. Note that the classes of $\mathcal{R}$ are countable and that $\alpha$ satisfies the cocycle equation: $\alpha(y, z) \alpha(x, y)=\alpha(x, z)$. Moreover, it is easy to see that one can choose a finite measure $\mu$ on $A$ such that the action map $(A \times G, \mu \times \lambda) \rightarrow(X, m)$ is locally measure preserving. By rescaling $\lambda$, we assume that $\mu$ is a probability measure. As $G$ is amenable and the action measure preserving, the equivalence relation $\mathcal{R}$ inherits two properties, called measure preserving and amenability (which we will not define here). It is therefore natural to develop entropy theory for quintuples of the form $(A, \mathcal{F}, \mu, \mathcal{R}, \alpha)$, where $\mathcal{R}$ is an amenable measure preserving equivalence relation with countable classes and $\alpha$ is a cocycle into an amenable group. This is described in Section 2.

For the second step, the following theorem is very useful:

Theorem 1.5 (see $[\mathrm{CFW}])$. Let $(X, \mathcal{F}, m)$ be a probability space. Let $\mathcal{R}$ be an amenable, measure preserving equivalence relation with countable classes. Then there exists a (measurable) transformation $T: X \rightarrow X$ such that for almost all $x, y \in X, x \mathcal{R} y$ iff there exists $n \in \mathbb{Z}$ such that $T^{n} x=y$.

Unfortunately, we cannot apply this theorem directly, since we have no control of the properties of $T$. The idea here (roughly, see [RW] for the original idea and Section 3 for our case) is to enlarge the space by multiplying it by an auxiliary action $Z$ and then relativising all our notions over $Z$. For example, a measure preserving action of $G$ on a space $(X, \mathcal{F}, m)$ is said to be relatively $C P E$ over $\mathcal{G}$, where $\mathcal{G}$ is an invariant sub-sigma-algebra, if for every partition $P$ which is not measurable with respect to $\mathcal{G}$, the relative entropy, $h(G, P \mid \mathcal{G})$, is positive. After this is done, one can choose $T$ to be measurable with respect to the sigma-algebra generated by the auxiliary action $Z$. It can be proved then that this $T$ is CPE over this sigma-algebra. The results are then deduced back from the theory of relatively K-transformations.

This work is a part of the author's MSc. thesis done at the Hebrew University under the supervision of Professor Benjamin Weiss. It is a pleasure to thank him for sharing with me his insights about mathematics in many discussions and for his encouragement.

\section{ENTROPY THEORY OF CROSS SECTIONS}

We start with two important examples of cross sections. In the following, $\lambda$ denotes the Haar measure of $G$ and $|\cdot|$ denotes the cardinality of a set.

Example 2.1. Suppose $\Gamma$ is a discrete amenable group. Let $X$ be a probability space and assume $\Gamma$ acts on $X$ in a measure preserving way. Then $X$ itself is a cross section of the action.

Example 2.2. Suppose $G$ is nondiscrete. Let $\Omega$ be the Poisson point process on $(G, \lambda)$. This means that $\Omega$ is a probability space whose points are discrete subsets

\footnotetext{
${ }^{1}$ In some cases (for example, when the group is discrete or is a nilpotent Lie group), assuming that the action is CPE and effective (no nontrivial element of the group acts trivially) implies that the action is free. However, this is not true in general.
} 
of $G$ such that for any subset $K \subset G$, the distribution of the random variable $|\omega \cap K|, \omega \in \Omega$, is Poisson with parameter $\lambda(K)$ and these random variables are independent for disjoint $K$ 's. Clearly, $G$ acts on $\Omega$ by translations, and this action is measure preserving. This action is called the Poisson action of $G$. The subset $S=\{\omega \in \Omega \mid 1 \in \omega\}$ is clearly a cross section.

Notation 2.3. If $A$ is a cross section as above, we say that $(A, \mathcal{F}, \mu, \mathcal{R}, \alpha)$ is the quintuple associated to $A$, and sometimes refer to it as the cross section. Such quintuples will be denoted by gothic letters $\mathfrak{A}, \mathfrak{B}$, etc.

Definition 2.4. For a function $f: A \rightarrow \mathbb{R}$ and a compact subset $F \subset G$ define

$$
f_{F}(x)=\frac{1}{|\{y \in A \mid \alpha(x, y) \in F\}|} \sum_{\{y \in A \mid \alpha(x, y) \in F\}} f(y) .
$$

We say that the mean ergodic theorem for $\mathfrak{A}$ holds if for any bounded Borel function $f: A \rightarrow \mathbb{R}$, the functions $f_{F}$ converge in probability to the constant function $\int_{A} f d \mu$ as $F$ becomes more and more invariant.

Theorem 2.5. The mean ergodic theorem holds for $\mathfrak{A}$ if and only if there exists a probability space $X$, an ergodic action of $G$ on $X$ and a cross section $S \subset X$ of this action such that the quintuple generated by this cross section is isomorphic to $\mathfrak{A}$.

Proof. (Sketch): Suppose $\mathfrak{A}$ is isomorphic to a cross section of a $G$ action on a space $X$. Given $f \in L_{\infty}(A)$, define $F \in L_{\infty}(X)$ by "fattening" $f$ : take a small identity neighborhood $U$ and let $F(u x)=f(x)$ where $x \in A$ and $u \in U$ and the representation is unique. Otherwise let $F$ be zero. Now use the mean ergodic theorem for amenable groups.

In the other direction, we take the Mackey range of the cocycle $\alpha$ which we now describe. Define an equivalence relation $\mathcal{S}$ on $A \times G$ by requiring $(x, g) \mathcal{S}(y, \alpha(x, y) g)$ for all $(x, y) \in \mathcal{R}$. Let $X=Y / \mathcal{S}$. $X$ is clearly a $G$ space and the set $\{(x, g) \mid$ $(\exists y \in A)(x, g) \mathcal{S}(y, 1)\}$ is a cross section isomorphic to $A$. The problem is to define a $G$ invariant probability measure on $X$. This is done by taking a fundamental domain $M \subset A \times G$ for $\mathcal{S}$ and showing that the mean ergodic theorem implies that every fundamental domain must have finite measure. After choosing a fundamental domain we project its measure to $X$.

Having a mean ergodic theorem, the second ingredient we need is a tiling lemma. Ignoring some technicalities, the lemma says that for every $\epsilon>0$ and every sufficiently invariant sequence $F_{1}, \ldots, F_{n} \subset G$, if $A \subset G$ is a finite set and if for every $a \in A$ the size of $a F_{k} \cap A$ is roughly $\lambda\left(F_{k}\right)$ for every $k$, then one can find a subset $B \subset A$ and a function $\sigma: B \rightarrow\{1, \ldots, n\}$ such that the sets $b F_{\sigma(b)} \cap A, b \in B$ form a quasi-tiling of $A$, i.e. these sets have small intersections (compared to their size) and cover $1-\epsilon$ of $A$. The lemma is proved in a similar way as in [L] or [W].

Given a mean ergodic theorem and a tiling lemma, we move on to define entropy. Assume $\mathfrak{A}$ is a quintuple satisfying the mean ergodic theorem. Let $P$ be a partition of $A$, where we think of a partition as a function from $A$ into some finite set.

Definition 2.6. Let $F \subset G$ be compact and $U \subset G$ be a neighborhood of 1 (we think of $F$ as being large and of $U$ as being small). Two points $x, y \in A$ are called $(\epsilon, F, U, P)$-close if there are subsets $X \subset\{z \in A \mid \alpha(x, z) \in F\}$ and $Y \subset\{z \in A \mid \alpha(y, z) \in F\}$ and a bijection $\psi: X \rightarrow Y$ such that

1. $|X| \geq(1-\epsilon)|\{z \in A \mid \alpha(x, z) \in F\}|$. 
2. $|Y| \geq(1-\epsilon)|\{z \in A \mid \alpha(y, z) \in F\}|$.

3. $P(z)=P(\psi(z))$ for every $z \in X$.

4. $\alpha(x, z) \in U \alpha(y, \psi(z))$ for every $z \in X$.

Stated less formally, $x$ and $y$ are close if the return times to $A$ which occur in $F$ and the colors $P$ assigns to them are roughly the same. We call a set an $(\epsilon, F, U, P)$-ball if every two points of it are $(\epsilon, F, U, P)$-close.

Definition 2.7. Let $\mathfrak{A}=(A, \mathcal{F}, \mu, \mathcal{R}, \alpha)$ be a cross section that satisfies the mean ergodic theorem. Let $P$ be a partition of $A, F \subset G$ compact, $U \subset G$ a neighborhood of the identity and $\epsilon>0$. Denote $n(\epsilon, F, U, P)$ the minimal size of a collection of $(\epsilon, F, U, P)$-balls whose union has measure larger than $1-\epsilon$. The entropy of $\mathfrak{A}$ with respect to the partition $P$ is

$$
h(\mathfrak{A}, P)=\lim _{\epsilon \rightarrow 0} \lim _{U} \lim _{F} \frac{1}{\lambda(F)} \log (n(\epsilon, F, U, P))
$$

where the limits are on $U$ 's getting smaller and $F$ 's getting more and more invariant.

The following is an analog of Abramov's formula:

Theorem 2.8. Assume $G$ is an amenable group with good entropy theory. Suppose $G$ acts on a probability space $X$ in a measure preserving way, and let $A \subset X$ be a cross section of this action. Then

$$
\sup _{P} h(G, P)=\sup _{Q} h(\mathfrak{A}, Q) .
$$

Remark 2.9. The multiplicative constant in Abramov's theorem disappeared because of our normalization of $\lambda$.

We also need to define entropy relative to a sub-sigma-algebra $\mathcal{G} \subset \mathcal{F}$ :

Definition 2.10. Let $(A, \mathcal{F}, \mu)$ be a probability space and let $\mathcal{G} \subset \mathcal{F}$ be a subsigma-algebra. Let $\mathcal{C}=\left\{C_{1}, \ldots, C_{N}\right\}$ be a collection of sets (not necessarily $\mathcal{G}$ measurable). The relative logarithmic size of $\mathcal{C}$ with respect to $\mathcal{G}$ is

$$
r l s(\mathcal{C} \mid \mathcal{G})=\int_{A} \log _{+}\left(\left|\left\{i \mid E\left(1_{C_{i}} \mid \mathcal{G}\right)(x)>0\right\}\right|\right) d \mu(x)
$$

where $\log _{+}(x)=\max \{\log (x), 0\}$.

Definition 2.11. Let $\mathfrak{A}, P, F, U, \epsilon$ be as before. Let $\mathcal{G} \subset \mathcal{F}$ be a sub-sigma-algebra such that $\mathcal{R}, \alpha$ are measurable with respect to $\mathcal{G} \otimes \mathcal{G}$. Let $n(\epsilon, F, U, P \mid \mathcal{G})$ be the minimal relative logarithmic size (with respect to $\mathcal{G}$ ) of a collection of $(F, U, P)$-balls that cover $1-\epsilon$ of $A$. Define the relative entropy as

$$
h(\mathfrak{A}, P \mid \mathcal{G})=\lim _{\epsilon \rightarrow 0} \lim _{U} \lim _{F} \frac{1}{\lambda(F)} n(\epsilon, F, U, P \mid \mathcal{G}) .
$$

Relating the definitions above to more classical definitions of entropy, we have the following:

Theorem 2.12. If $\Gamma$ is a discrete amenable group acting on a probability space $X$, then $X$ itself is a cross section for this action. If $\mathfrak{X}$ denote the quintuple of this cross section, then $h(\mathfrak{X}, P \mid \mathcal{G})=h(\Gamma, P \mid \mathcal{G})$ for every partition $P$ and every invariant sub-sigma-algebra $\mathcal{G} \subset \mathcal{F}$. 
Theorem 2.13. Let $G$ be nondiscrete. Assume that the action of $G$ on $X$ is CPE. Let $\Omega$ be the Poisson action and let $S \subset \Omega$ be a cross section. Then $X \times S$ is a cross section of the diagonal action of $G$ on $X \times \Omega$, and it is relatively CPE over the second coordinate sigma-algebra.

Another useful fact is the following:

Theorem 2.14. Let $(A, \mathcal{F}, m)$ be a probability space. Let $\mathcal{R}$ be a discrete equivalence relation on $X$ and let $\alpha: \mathcal{R} \rightarrow G, \beta: \mathcal{R} \rightarrow H$ be two cocycles such that $G, H$ are amenable groups with good entropy theory and the two quintuples $\mathfrak{A}=(A, \mathcal{F}, m, \mathcal{R}, \alpha)$ and $\mathfrak{B}=(A, \mathcal{F}, m, \mathcal{R}, \beta)$ satisfy the mean ergodic theorem. Then for every sub-sigma-algebra $\mathcal{G} \subset \mathcal{F}$ such that both $\alpha$ and $\beta$ are $\mathcal{G}$-measurable and for every partition $P$ of $A$ we have $h(\mathfrak{A}, P \mid \mathcal{G})=h(\mathfrak{B}, P \mid \mathcal{G})$.

These theorems allow us to transfer results from the classical ergodic theory to ergodic theory of more general amenable groups. An example is given in the next section.

\section{Proof of Theorem 1.4}

In this section we combine the material of Section 2 with the methods of [DG] to sketch the proof of the second part of Theorem 1.4. A similar kind of combination of Section 2 and $[\mathrm{RW}]$ will prove the first part of Theorem 1.4. Also, we only prove the theorem for the class of groups with zero self-entropy, which is a subclass of the groups with good entropy theory (see [OW] for the definition; we note that this class contains all nilpotent Lie groups). In the general case of groups with good entropy theory, Lemma 3.4 requires a different proof. We can also assume $G$ is nondiscrete.

We start with an action of $G$ on $X$ which is CPE. Let $\Omega$ be the Poisson action of $G$ and let $S \subset \Omega$ be a cross section. We further require that the cross section is uniformly discrete, that is, there exists a neighborhood $U$ of the identity of $G$ such that $\alpha(x, y) \in U \Rightarrow \alpha(x, y)=1$. It is easy to construct such a cross section. Consider the product space $X \times \Omega$ and the diagonal action $G$ on it. By Theorem 2.13 the cross section $X \times S \subset X \times \Omega$ is CPE over the second coordinate sigmaalgebra, which we denote $\mathcal{F}_{\Omega}$. Denote the equivalence relation on $S$ by $\mathcal{S}$. By Theorem 1.5 there exists a transformation $T: S \rightarrow S$ that generates $\mathcal{S}$. Define $\bar{T}: X \times S \rightarrow X \times S$ by $\bar{T}(x, s)=(\alpha(s, T s) x, T s)$. It is easy to show that $\bar{T}$ generates the equivalence relation on $X \times S$ induced by the diagonal action and that $\bar{T}$ is $\mathcal{F}_{\Omega}$-measurable. By Theorems 2.12 and $2.14, \bar{T}$ is relatively $\mathrm{CPE}$ over $\mathcal{F}_{\Omega}$. It is known (see [KL]) that there exists a sub-sigma-algebra $\mathcal{E} \subset \mathcal{F}_{X} \otimes \mathcal{F}_{\Omega}$ such that

$$
\mathcal{F}_{\Omega}=\bigcap_{n} \bigvee_{k=-\infty}^{n} T^{k}(\mathcal{E}) \subset \cdots \subset \bigvee_{k=-\infty}^{0} T^{k}(\mathcal{E}) \subset \cdots \subset \bigvee_{k=-\infty}^{\infty} T^{k}(\mathcal{E})=\mathcal{F}_{X} \otimes \mathcal{F}_{\Omega}
$$

Let $\phi_{i}$ be an orthonormal basis of $L_{2}\left(X \times S, \bigvee_{-\infty}^{1} T^{k}(\mathcal{E})\right) \ominus L_{2}\left(X \times S, \bigvee_{-\infty}^{0} T^{k}(\mathcal{E})\right)$. Recall that the cross section $X \times S$ is uniformly discrete. If $V$ is open and satisfies $V^{2} \subset U$, define the functions $\Phi_{i}^{V} \in L_{2}(X \times \Omega)$ by $\Phi_{i}^{V}(v p)=\phi(p)$ if $p \in X \times S$ and $v \in V$, and let $\Phi_{i}^{V}$ be zero otherwise.

Lemma 3.1. For every $V, W$ such that $V^{2}, W^{2} \subset U$, for every $i, j$ and every $g \in G$ we have

$$
\left\langle g \Phi_{i}^{V}, \Phi_{j}^{W}\right\rangle=\delta_{i j} \lambda(g V \cap W)=\delta_{i j}\left\langle g 1_{V}, 1_{W}\right\rangle .
$$


Lemma 3.2. The collection of $\Phi_{i}^{V}$ generates $L_{2}\left(X \times \Omega, \mathcal{F}_{X} \otimes \mathcal{F}_{\Omega}\right) \ominus L_{2}\left(X \times \Omega, \mathcal{F}_{\Omega}\right)$.

Corollary 3.3. The $G$-module $L_{2}(X)$ is contained in a countable orthogonal sum of $L_{2}(G)$ 's.

Proof. If we denote the indicator function of $V$ by $1_{V}$, Lemma 3.1 implies that for every $i$, the map $g 1_{V} \rightarrow g \Phi_{i}^{V}$ extends to an isometry from $\operatorname{span}\left\{g 1_{V} \mid g \in G\right.$, $\left.V^{2} \subset U\right\}=L_{2}(G)$ into $L_{2}(X \times \Omega)$ and moreover, the different images are orthogonal. Lemma 3.2 implies that the direct sum of the images (for all $i$ 's) is total. Therefore $L_{2}(X \times \Omega) \ominus L_{2}(\Omega)$ is a countable direct sum of $L_{2}(G)$ 's. However, $L_{2}(X)$ is a submodule of it.

Lemma 3.4. $L_{2}(X)$ contains a countable orthogonal sum of $L_{2}(G)$ 's.

Proof. By [OW], $X$ has a Bernoulli factor $Y$ ( $Y$ is called Bernoulli if it is a factor of the Poisson action of $G$ ). By the isomorphism theorem in [OW], there are Bernoulli actions $Z_{1}, Z_{2}$ such that $Y=Z_{1} \times Z_{2}$. It is easy to generalize Theorem 2.13 for Bernoulli actions (instead of Poisson actions). Doing that, the arguments in this section show that $L_{2}(Y) \ominus L_{2}\left(Z_{1}\right)$ is a countable orthogonal sum of $L_{2}(G)$ 's. We finish since $L_{2}(Y)$ is a submodule of $L_{2}(X)$.

The proof of Theorem 1.4 is completed using a Cantor-Bernstein type argument.

\section{REFERENCES}

$[\mathrm{CFW}]$ A. Connes, J. Feldman, and B. Weiss, An amenable equivalence relation is generated by a single transformation, Ergodic Theory Dynamical Systems 1 (1981), no. 4, 431-450 (1982). MR0662736 (84h:46090)

[DP] A. I. Danilenko and K. K. Park, Generators and Bernoullian factors for amenable actions and cocycles on their orbits, Ergodic Theory and Dynamical Systems 22 (2002), no. 6, 1715-1745. MR1944401 (2004f:37006)

[DG] A. H. Dooley and V. Ya. Golodets, The spectrum of completely positive entropy actions of countable amenable groups, J. Funct. Anal. 196 (2002), no. 1, 1-18. MR1941988 (2003m:37006)

[KL] B. Kamiński and P. Liardet, Spectrum of multidimensional dynamical systems with positive entropy, Studia Math. 108 (1994), no. 1, 77-85. MR1259025 (94m:28035)

[L] E. Lindenstrauss, Pointwise theorems for amenable groups, Invent. Math. 146 (2001), no. 2, 259-295. MR.1865397 (2002h:37005)

[OW] D. S. Ornstein and B. Weiss, Entropy and isomorphism theorems for actions of amenable groups, J. Analyse Math. 48 (1987), 1-141. MR0910005 (88j:28014)

[RS] V. A. Rohlin and Ja. G. Sinal̆, The structure and properties of invariant measurable partitions (Russian), Dokl. Akad. Nauk SSSR 141 (1961), 1038-1041. MR0152629 (27:2604)

[RW] D. J. Rudolph and B. Weiss, Entropy and mixing for amenable group actions, Ann. of Math. (2) 151 (2000), no. 3, 1119-1150. MR1779565 (2001g:37001)

[S] Ja. G. Sină̌, Dynamical systems with countable Lebesgue spectrum (Russian), Izv. Akad. Nauk SSSR Ser. Math. 25 (1961), 899-924. MR0148852 (26:6356)

[W] B. Weiss, Actions of amenable groups, Topics in Dynamics and Ergodic Theory, LMS Lect. Note Series 310, 226-262. MR 2052281 (2005d:37008)

Department of Mathematics, Hebrew University of Jerusalem, Israel

E-mail address: anir@math.huji.ac.il 